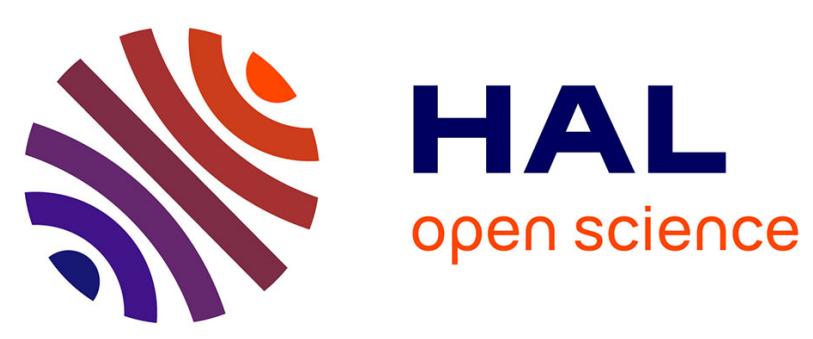

\title{
Éthique et justice dans les recherches participatives ancrées dans les communautés. Le cas d'une enquête écosystémique en Amazonie \\ Nicolas Lechopier
}

\section{- To cite this version:}

Nicolas Lechopier. Éthique et justice dans les recherches participatives ancrées dans les communautés. Le cas d'une enquête écosystémique en Amazonie. Éthique publique - revue internationale d'éthique sociétale et gouvernementale , 2010, 12 (1), pp.201-223. 10.4000/ethiquepublique.183 . halshs-00840370

\section{HAL Id: halshs-00840370 \\ https://shs.hal.science/halshs-00840370}

Submitted on 2 Jul 2013

HAL is a multi-disciplinary open access archive for the deposit and dissemination of scientific research documents, whether they are published or not. The documents may come from teaching and research institutions in France or abroad, or from public or private research centers.
L'archive ouverte pluridisciplinaire HAL, est destinée au dépôt et à la diffusion de documents scientifiques de niveau recherche, publiés ou non, émanant des établissements d'enseignement et de recherche français ou étrangers, des laboratoires publics ou privés. 


\title{
Éthique publique
}

vol. $12, \mathrm{n}^{\circ} 1$ (2010)

Responsabilité sociale et éthique de la recherche

Nicolas Lechopier

\section{Éthique et justice dans les recherches participatives ancrées dans les communautés. Le cas d'une enquête écosystémique en Amazonie}

\begin{abstract}
Avertissement
Le contenu de ce site relève de la législation française sur la propriété intellectuelle et est la propriété exclusive de l'éditeur.

Les œuvres figurant sur ce site peuvent être consultées et reproduites sur un support papier ou numérique sous réserve qu'elles soient strictement réservées à un usage soit personnel, soit scientifique ou pédagogique excluant toute exploitation commerciale. La reproduction devra obligatoirement mentionner l'éditeur, le nom de la revue, l'auteur et la référence du document.

Toute autre reproduction est interdite sauf accord préalable de l'éditeur, en dehors des cas prévus par la législation en vigueur en France.
\end{abstract}

\section{revues.org}

Revues.org est un portail de revues en sciences humaines et sociales développé par le Cléo, Centre pour l'édition électronique ouverte (CNRS, EHESS, UP, UAPV).

\author{
Référence électronique \\ ethiquepublique.183 \\ Éditeur : Éditions Nota bene \\ http://ethiquepublique.revues.org \\ http://www.revues.org \\ Document accessible en ligne sur : http://ethiquepublique.revues.org/183 \\ Ce document est le fac-similé de l'édition papier. \\ Tous droits réservés
}

Nicolas Lechopier, «Éthique et justice dans les recherches participatives ancrées dans les communautés. Le cas d'une enquête écosystémique en Amazonie », Éthique publique [En ligne], vol. 12, n 1 | 2010, mis en ligne le 10 mai 2011, consulté le 10 octobre 2012. URL : http://ethiquepublique.revues.org/183 ; DOI : 10.4000/ 


\title{
ÉTHIQUE ET JUSTICE \\ DANS LES RECHERCHES \\ PARTICIPATIVES ANCRÉES \\ DANS LES COMMUNAUTÉS. \\ LE CAS D'UNE ENQUETTE \\ ÉCOSYSTÉMIQUE EN AMAZONIE
}

\author{
NICOLAS LECHOPIER \\ Université Lyon 1
}

Résumé: Depuis les années 1970, les recherches participatives ancrées dans les communautés ont peu à peu gagné du crédit dans le champ des recherches appliquées, entraînant des mutations épistémiques et éthiques dans les pratiques de recherche avec des participants humains. Le présent article s'appuie sur un exemple de recherche «écosystémique» en santé environnementale conduite en partenariat entre des chercheurs universitaires (Canada et Brésil) et des habitants des rives d'un fleuve de l'Amazonie brésilienne, le Tapajós. Cet exemple illustre les tensions qui affectent le cadre conceptuel dans lequel sont habituellement posées les questions d'éthique de la recherche avec des participants humains, notamment les principes cardinaux tirés du rapport Belmont, et tout particulièrement ceux de bienfaisance et de justice. Ce genre de pratiques scientifiques implique un redéploiement de la responsabilité des chercheurs, entre recherche et action. 


\section{INTRODUCTION}

Les recherches participatives ancrées dans les communautés (en anglais, community-based participatory research; ci-dessous désignées $R P C$ ) forment une famille de méthodes réunies autour de pratiques et de valeurs. Les RPC constituent un dispositif d'enquête reposant sur l'association entre des chercheurs et des groupes concernés (habitants d'une localité, travailleurs d'une entreprise, élèves d'une école, pouvoirs publics, etc.) autour d'un problème de recherche qui fait sens pour les uns et les autres. Selon les cas, on appelle ces groupes concernés " communautés ", «société civile» ou «le public»(Mohan, 1999). Ces recherches sont "participatives » puisque les groupes concernés vont participer au déroulement de la recherche, au moment de sa mise en œuvre et de l'évaluation pratique de ses résultats, mais aussi le cas échéant, en amont, au moment de définir les problèmes de recherche et de formuler les hypothèses. Ces recherches ont pour but explicite de réaliser une intégration de la connaissance et de l'action, en ayant en vue le bénéfice de tous les partenaires et pas seulement celui des chercheurs (Israel et al., 1998).

Les méthodes participatives représentent une approche qui diffère à de nombreux égards des modèles traditionnels de la recherche. Dans un premier temps, après avoir souligné trois caractéristiques qui les distinguent des méthodologies traditionnelles privilégiant la position de distanciation du chercheur par rapport à l'objet, j'insisterai sur le fait que les RPC produisent des tensions sur le cadre éthique le plus couramment employé dans la réflexion sur la recherche conduite auprès des êtres humains, fondé sur des principes. Dans un second temps, je présenterai une étude de cas illustrant certaines de ces tensions et montrant la nécessité d'une clarification de la sphère de responsabilité des chercheurs.

\section{LES RECHERCHES PARTICIPATIVES : QUALITÉS ET FRAGILITÉS}

\section{PARTICULARITÉS SUR LE PLAN MÉTHODOLOGIQUE}

Les RPC présentent au moins trois caractéristiques qui font leur caractère propre. La première est que les RPC abordent les problèmes de recherche sans en réduire la complexité et le caractère multidimensionnel. Ces 
recherches ancrées dans les communautés sont fortement contextualisées, et les connaissances produites sont réputées être pertinentes, significatives et localement appropriées (Green, Daniel et Novick, 2001). L'implication des participants à la fois suppose et permet de mieux comprendre comment le phénomène (par exemple, une épidémie, une pollution ayant des répercussions sur la santé, etc.) s'inscrit dans les dynamiques environnementales, économiques et sociales qui le traversent (Israel et al., 1994).

Une seconde caractéristique introduit une rupture par rapport au modèle classique de la recherche: alors que la vision traditionnelle de la pratique scientifique s'est construite autour d'une dichotomie entre savoir et pouvoir, production de connaissance et applications, sciences et politique, les RPC se donnent souvent pour objectif de contribuer au renforcement de la puissance d'agir des personnes concernées (empowerment). L'émancipation de groupes socialement et économiquement dominés peut devenir l'un des buts de la recherche scientifique - et non pas simplement une «retombée » ou un simple effet secondaire. Les connaissances produites par les RPC sont en ce sens explicitement intéressées. Elles devraient permettre que les communautés dominées renforcent leurs capacités à agir sur les problèmes qui les affectent. Sans prétendre dessiner ici la généalogie complexe de cet engagement de la recherche sur le terrain politique, on peut mentionner au moins deux sources: les recherches-actions conduites avec les travailleurs dans leurs milieux de travail par Kurt Lewin dans les années 1940 (Lewin, 1946; Adelman, 1993) et les pratiques d'éducation populaire élaborées par Paulo Freire dans les années 1960 et 1970 (Freire, 1974).

Enfin, les RPC s'adossent à une exigence de réflexivité. La participation des communautés n'a pas une valeur simplement instrumentale, au sens où elle rendrait plus aisé le recueil de données ou l'acceptation des résultats. La participation conduit aussi à mettre en question explicitement l'attitude des parties prenantes, la valeur d'un problème de recherche, l'utilité en pratique des connaissances générées, etc. Comme y insistent plusieurs auteurs, un idéal d'équité règle les rapports entre les chercheurs et les communautés (Minkle et al., 2003), ce qui implique de prendre le contrepied d'une domination de type paternaliste entre chercheurs et sujets (Muller-Mirza, 2009), en particulier dans les recherches impliquant des «chercheurs» du Nord et des «partenaires» du Sud. Sans effacer les 
différences entre ces différents partenaires sur les plans du symbolique et des compétences, les RPC pourraient être le lieu d'une relation plus égalitaire et horizontale entre chercheurs et participants.

Ces caractéristiques des RPC ont pour conséquence de modifier la posture traditionnelle du chercheur, position de surplomb, maintenant une distance par rapport à l'objet. Tout en restant compatibles avec les principales valeurs épistémiques propres à toute recherche de connaissance vérifiée (adéquation empirique, cohérence interne, etc.), ces formes de recherche se démarquent de celles qui sont centrées sur l'emploi de modèles prédictifs, décontextualisés ou réductionnistes ${ }^{1}$. Ce décalage par rapport aux standards dominants est renforcé par des particularités procédurales. Par exemple, le plan d'enquête des RPC n'est souvent pas entièrement fixé en amont. Il peut être amené à se transformer en cours de route, notamment à partir des interactions entre chercheurs et communautés. De plus, les données recueillies par les chercheurs incluent, autant que possible, les valeurs des participants. Dans le cas des approches collaboratives, ce sont les participants eux-mêmes qui collectent les données. Cela peut générer pour la recherche des informations plus riches, mais cela augmente aussi le risque de biais difficiles à contrôler. On retrouve ici la question plus générale de la pluralité des critères épistémiques et celle du clivage entre les approches qualitatives et quantitatives (Strauss et Corbin, 2008). Les commissions de financement et les pairs chargés d'évaluer la qualité de ces projets doivent se forger des critères d'évaluation qui prennent en compte ces particularités. Cela n'est pas simple, même si ce genre de méthodes est de mieux en mieux accepté, au moins en Amérique du Nord (Turckheim, Hubert et Cerf, 2009).

\section{TENSIONS TOUCHANT AU CADRE DE L'ÉVALUATION ÉTHIQUE}

Aborder la dimension de l'évaluation éthique des RPC suppose de prendre en compte plusieurs points de vue, et pas seulement l'attitude des chercheurs à l'égard des participants. Du côté des chercheurs, conduire ce genre d'enquête sous-tend inévitablement un engagement personnel im-

1. Pour une analyse de la distinction entre valeurs cognitives et valeurs extra-cognitives, voir Lacey (2005). 
portant, puisqu'il leur faut consacrer beaucoup de temps dans des réunions communautaires, savoir négocier dans un contexte où la dimension politique peut s'avérer prégnante, manifester un sens de l'équité afin d'instaurer et de conserver de bons rapports avec les participants, etc. On a parlé à cet égard d'une "éthique de l'implication», caractéristique de la démarche des chercheurs engagés dans ce genre de recherches (Shore, 2006 : 11). Cette implication se comprend aussi comme une implication personnelle, humaine, relationnelle.

Du côté des communautés, les RPC ne sont rien d'anodin. Occasions pour les participants d'entrer dans des interactions sociales plus ou moins durables avec des intervenants extérieurs, ces recherches peuvent faire bouger les équilibres sociaux établis. On retrouve ici le constat, bien connu des chercheurs en sciences sociales, selon lequel tout processus de recherche - surtout s'il se fait avec des chercheurs qui ne sont pas membres de la communauté concernée - introduit d'emblée des perturbations qui peuvent avoir des retentissements durables (Minkler, 2004). Dans ces interactions, des rapports de domination symbolique peuvent être reconduits, même lorsque les chercheurs témoignent de «bonne volonté». La fragilité et la vulnérabilité des personnes concernées par le problème faisant l'objet de la recherche appellent, selon certains, une éthique du "prendre soin » (care) lucide par rapport aux conditions contextuelles dans lesquelles les relations se développent (Thiery et Cerf, 2009: 44).

L'adéquation entre cette dimension relationnelle et les cadres courants de l'éthique de la recherche avec des sujets humains n'est pas évidente. L'approche traditionnelle pour l'évaluation de cette éthique a été clarifiée au milieu des années 1970, en Amérique du Nord, avec notamment le rapport de synthèse de la commission nationale américaine (1984) (le rapport Belmont, 1978). Celui-ci mettait en lumière un triple référentiel qui a donné lieu au courant du «principisme" (principism) et qui fait référence à trois principes éthiques fondamentaux: la bienfaisance, le respect des sujets et la justice. On les rappellera ici brièvement. Le premier de ces principes exige de ne pas causer de torts aux participants et à la société, et le cas échéant de promouvoir leur bien-être. Le deuxième demande de respecter la liberté des sujets et leur capacité de délibération. Le troisième principe demande une juste distribution du fardeau et des bénéfices de la recherche. Ces trois principes ne constituent pas des règles 
opérationnelles précises ${ }^{2}$, mais ils sont censés les inspirer et sont d'ailleurs aujourd'hui encore à la base de la plupart des recommandations faites aux chercheurs qui conduisent des recherches avec des participants humains ${ }^{3}$.

Nancy Shore (2006) a conduit une riche enquête sur la pertinence de ces principes, du point de vue des chercheurs conduisant des RPC. Selon elle, les recherches participatives requièrent des aménagements importants dans leur interprétation pratique, sans toutefois les invalider en tant que tels. En premier lieu, on note que le principe de bienfaisance, à la lumière des RPC et contrairement à d'autres recherches cliniques et surtout épidémiologiques, ne saurait se réduire à la non-malfaisance : les communautés participantes doivent clairement trouver un bénéfice dans la recherche et à son issue. L'approche participative implique donc de laisser clairement une place à l'évaluation du processus par les participants eux-mêmes, ce qui n'est pas sans soulever des difficultés, surtout dans un contexte multiculturel. Les chercheurs interrogés par Shore considèrent d'ailleurs que ces résultats ne s'interprètent pas seulement en fonction d'indicateurs épidémiologiques ou socioéconomiques (incidence de la maladie, revenus moyens, qualité de vie, etc.), mais aussi en fonction du renforcement de la puissance d'agir des communautés participantes.

En deuxième lieu, le principe de respect des sujets, dans les RPC, apparaît moins comme une étape déterminée dans le processus de recherche (par exemple, le moment de l'obtention du "consentement informé») que comme un processus relationnel, entretenu dans la durée, à la faveur d'un climat de confiance mutuelle. Les chercheurs insistent ainsi sur l'authenticité du participatif: la participation doit être autre chose qu'un simple instrument servant à renforcer l'acceptation sociale d'une proposition définie en amont. Elle devrait permettre de faire en sorte que les personnes contribuent effectivement à affiner la recherche et à la rendre plus pertinente. Ce point est particulièrement délicat, dans la mesure où le

2. Sur la différence entre "principe » et « règles », voir Macklin (1999). Pour une défense illustrée du principisme spécifié, voir l'article de Massé (2003).

3. Au Canada, l'introduction du texte normatif principal cite le rapport Belmont comme une source importante. Voir l'Énoncé de politique des trois conseils: éthique de la recherche avec des êtres humains, édicté par les Instituts de recherche en santé du Canada, le Conseil de recherches en sciences naturelles et en génie du Canada et le Conseil de recherches en sciences humaines du Canada, 1998 (rapport modifié en 2000, 2002 et 2005). 
terme participation, utilisé à propos de pratiques scientifiques très hétérogènes (de la simple consultation à la co-construction), peut gêner l'analyse lucide des rapports qui se jouent précisément dans le rapport entre chercheurs et participants.

En troisième lieu, l'exigence de justice distributive formulée dans le troisième principe subit un déplacement significatif. Dans le rapport Belmont, ce principe était formulé en ayant à l'esprit les risques d'exploitation par les chercheurs de sujets vulnérables ou déconsidérés (experimentum in corpore vili) (Chamayou, 2008). De fait, en adoptant une démarche participative et à condition qu'elles soient honnêtement conduites, les RPC ne semblent pas présenter de risque d'injustice distributive : le fardeau de la recherche et ses bénéfices attendus sont rattachés, en principe, aux mêmes personnes. De plus, le parti pris des RPC en faveur de l'autonomie et du renforcement de la puissance d'agir des participants positionne ce genre de recherche comme un moyen pour corriger des injustices préexistantes, en s'attaquant aux inégalités de condition. Certains auteurs tendent ainsi à réinterpréter le principe, énoncé dans le rapport Belmont comme une «justice distributive», dans le sens d'une «justice relationnelle» (King, Henderson et Stein, 1999). On verra cependant à partir du cas exposé en détail ci-dessous que la question de la justice reste une question délicate aux frontières de la sphère de responsabilité des chercheurs.

\section{LE PROBLÈME DE LA DISTINCTION ENTRE RECHERCHE ET PRATIQUE}

Si les principes à la base de l'évaluation éthique de la recherche avec les êtres humains peuvent ainsi trouver une interprétation propre aux RPC, il faut noter que ce cadre général est construit sur un présupposé que Shore ne remarque que de façon incidente alors qu'il a indéniablement une certaine importance. L'introduction du rapport Belmont préconise en effet de distinguer la «recherche» de la «pratique »: «Il est important de distinguer entre recherche biomédicale [...] d'une part, et pratique thérapeutique courante d'autre part, dans le but de connaître quelles activités doivent subir une révision pour la protection des sujets humains" (Commission nationale américaine, 1984). Cette distinction préalable entre pratique et recherche est, dans les comités de révision de la recherche, généralement tenue pour acquise : la recherche se distingue de 
la pratique courante en ce qu'elle constitue une activité visant principalement à produire des connaissances. Pourtant, il arrive fréquemment qu'on ait des difficultés à démêler ce qui relève de l'un ou l'autre genre d'activité - en particulier dans le domaine de la santé publique et des sciences sociales (Ouellet-Dubé, 1979). Or, cette distinction a des retentissements sérieux quant à la définition du domaine de responsabilité du chercheur à l'égard des participants. Elle signifie que le chercheur qui conduit des recherches avec lêtre humain est responsable des actes qu'il pratique en tant que chercheur et de leurs conséquences directes et indirectes, mais il n'est pas directement responsable de tout le contexte (social, politique, etc.) dans lequel sa recherche s'inscrit. Les principes éthiques valent, pour ainsi dire, sous une clause caeteris paribus: ce sont les actes pratiqués au nom de la recherche qui comptent d'un point de vue éthique et non pas les conditions contextuelles, indépendantes de l'acte de recherche.

Cette distinction soulève une difficulté pour les RPC puisque, en intégrant étroitement recherche et action, production de connaissance et intervention, elles brouillent cette distinction préalable posée dans l'approche principaliste énoncée par le rapport Belmont. Comment se définit la sphère de responsabilité des chercheurs lorsque la recherche est délibérément contextualisée, ancrée dans la communauté, ouverte à la participation? De quoi précisément sont responsables éthiquement les chercheurs en RPC ? Ces questions orienteront l'étude de cas suivante.

\section{LE CAS D'UNE RECHERCHE ÉCOSYSTÉMIQUE SUR LA POLLUTION AU MERCURE EN AMAZONIE}

Au début des années 2000, Jean Lebel a théorisé l'approche écosystémique dans la recherche sur les interactions entre santé humaine et environnement (Lebel, 2003). Cette approche peut être résumée comme suit. La notion d'écosystème, appliquée au domaine de la santé humaine, conduit à un élargissement des concepts de santé et de maladie: la santé n'est pas absence de maladies mais plutôt la "participation harmonieuse aux ressources de l'environnement, qui dispose les individus à un plein exercice de leurs fonctions et de leurs aptitudes»(Dansereau, 2003: 8). L'approche écosystémique est anthropocentrique (ce sont les besoins humains qui sont pris comme référence pour penser l'écosystème), mais les problèmes de 
santé sont contextualisés (on prend en compte les multiples interactions entre les humains et leur milieu). L'un des exemples typiques de ce genre d'approche - qui est en aussi la source majeure d'inspiration pour Lebel, qui a fait sa thèse dans ce cadre - est le projet Caruso. Cette recherche, dont je détaillerai un épisode ci-dessous, a été conduite de 1994 à 2008 par des chercheurs canadiens et brésiliens avec les habitants des rives du fleuve Tapajós, dans l'État du Pará, en Amazonie brésilienne ${ }^{4}$.

Depuis plusieurs décennies, on savait que les habitants de ces régions sont contaminés par le mercure à doses relativement faibles en raison de leur consommation de poissons. Cette contamination a des répercussions sur leur santé en augmentant le risque d'avoir des problèmes neurologiques, fonctionnels et cardiovasculaires (Passos et Mergler, 2005 ; Lucotte et al., 2005). Au début des années 1990, un médecin cardiologue de Santarém, Fernando Branches, donnait de temps en temps des consultations à Brasília Legal, village des bords du Tapajós. À partir de certaines observations cliniques inquiétantes, il a soulevé la question du rôle du mercure dans l'état de santé de la population et a noué des relations avec des chercheurs canadiens afin de conduire des recherches ${ }^{5}$. La «commande de recherche » est issue d'une personnalité locale (Branches) liée à des chercheurs qui y ont vu un terrain de recherche intéressant. En effet, contrairement à d'autres endroits du monde où les pollutions au mercure sont étudiées (notamment à Minamata au Japon), il semble être dans cette région amazonienne le seul métal présent dans l'environnement affectant la santé humaine, ce qui rend le terrain particulièrement fécond pour des chercheurs souhaitant comprendre l'impact propre au mercure.

Au milieu des années 1990, les chercheurs du projet Caruso ont découvert que la présence de ce métal lourd dans l'environnement nétait pas seulement due à l'activité d'extraction d'or-les mines furent très nombreuses aux bords du fleuve il y a quelques décennies -, mais aussi aux activités agricoles fondées sur le brûlis: le mercure présent naturellement

\footnotetext{
4. Les détails sur le projet CARUSO, financé par le Centre de recherche sur le développement international, peuvent être trouvés à l'adresse suivante: www.unites.uqam.ca/gmf/caruso/caruso.htm. 5. Fernando Branches est l'un de ceux qui ont alerté la communauté mondiale sur les relations entre mercure et dommages cardiovasculaire. Il est aussi l'auteur d'un test (le BAMT, Branches Alternate Movement Task), un test de coordination motrice qui est bien corrélé en ce qui a trait au mercure. Sur Branches, voir Lebel (2002).
} 
dans le sol à l'état inorganique est déversé dans l'eau du fleuve par l'érosion générée par la déforestation, puis transformé en une substance organique assimilable par les poissons, et par les humains qui les consomment (Roulet et al., 1999). Pour découvrir l'origine de la contamination au mercure, les chercheurs ont eu besoin du concours de multiples disciplines parmi les sciences de la terre, la biologie et les sciences sociales, permettant de prendre en compte les multiples dimensions du problème. Les chercheurs ont peu à peu reconstitué un réseau d'interactions - un écosystème - incluant le cycle biochimique de transformation du mercure, les pratiques agricoles fondée sur le brûlis, la pêche donnant la préférence à certaines espèces, les vagues de colonisation de ces terres par des paysans issus d'autres régions du pays, etc. La pollution au mercure et les risques pour la santé étaient ainsi replacés dans une analyse systémique, située, contextualisée, attentive à la façon dont les individus interagissent entre eux autour de ce problème.

Alors que le projet était initialement centré sur l'évaluation de la contamination et des interactions entre pollution et activités humaines (approche descriptive et explicative), les habitants ont demandé à travailler sur des mesures permettant de réduire les effets de cette pollution. Dans la seconde phase, la recherche s'est progressivement orientée vers l'étude de mesures préventives. À la suggestion et avec l'aide opérationnelle d'un groupe de femmes qui ont tenu patiemment un recueil scrupuleux de leurs menus, les chercheurs ont établi par des études épidémiologiques participatives que la consommation de fruits tropicaux pouvait avoir des conséquences favorables et réduire le niveau de contamination au mercure (Passos, 2009). Au cours et au terme de ces recherches, les habitants ont reçu des informations claires et accessibles concernant les causes de la contamination au mercure (notamment le rôle de l'agriculture sur brûlis) et des préconisations alimentaires réalistes, permettant de réduire les effets du mercure sur la santé à long terme.

Outre des réunions d'information, différents outils ont été mis en place pour favoriser la participation des communautés: une sociologue de la région a mis en place des focus groups (Gaspar, 2003) pour évaluer qualitativement ces mesures préventives, et notamment les changements relatifs aux habitudes alimentaires, ce qui a complété les données épidémiologiques quantitatives. Des outils de modélisation des réseaux sociaux ont 
également permis de décrire les circuits par lesquels les habitants des communautés riveraines du Tapajós pouvaient être efficacement et équitablement associés à ces connaissances sur la pollution au mercure. Ces différents outils ont aidé à désigner des leaders d'opinion et à déterminer le rôle essentiel des femmes pour ce qui concerne les questions d'éducation à l'alimentation et à la santé.

Comme on le voit, le projet Caruso s'est mis en place, pas à pas, à l'initiative des chercheurs, avec un souci constant de maintenir de bonnes relations avec les communautés. Les riverains du Tapajós, possiblement blasés par les différents projets (gouvernementaux, universitaires ou humanitaires) conduits jusque-là dans leur région, ont entretenu avec les chercheurs des relations assez riches, notamment grâce à de multiples réunions d'information et de consultation. Peu à peu, la participation des communautés dans le projet Caruso est passée d'un mode «consultatif» à un mode plus «collaboratif» (Mertens et al., 2005).

\section{LE PROJET VISION ET LA PRESCRIPTION DE LUNETTES}

L'une des actions de recherche réalisées par les chercheurs de l'équipe santé du Caruso, le projet Vision, mérite une analyse plus détaillée. Le choix de ce cas ne signifie pas qu'il doit être considéré comme l'épisode le plus «grave» ou «critique» dans l'histoire, assez longue, des relations entre chercheurs et participants dans le Tapajós. Ce choix découle d'un événement vécu sur le terrain qui m'a conduit à reconstituer - essentiellement sur la base de témoignages des chercheurs eux-mêmes - un épisode révélateur des ambivalences de la relation entre chercheurs et participants ${ }^{6}$.

\footnotetext{
6. Le cas ci-dessous a été reconstitué sur la base de témoignages recueillis au mois de mai 2009, à l'occasion d'un séjour de recherche consacré au projet qui a pris la relève du CARUSO, le projet Poor Land Use, Poor Health, qui traite des interactions entre pollution au mercure, agriculture fondée sur les brûlis et maladie de Chagas et comprend un volet d'expérimentation avec les villageois de systèmes agroforestiers. Il se trouve que, lors d'une visite des chercheurs à des villageois de la région, ces derniers ont demandé de façon insistante si le projet incluait une mise à disposition de lunettes. Les chercheurs ont eu peine à expliquer que cela n'était pas inclus dans leur projet. À partir de cet événement, j’ai recueilli des témoignages sur son contexte auprès de chercheurs, tant de l'équipe santé, directement concernée, que de l'équipe environnement, ainsi que de participants au projet rencontrés sur place et d'autres témoins (agent communautaire de santé, pêcheurs, personnel de bord du bateau affrété par l'équipe de recherche, etc.).
} 
Le contexte scientifique de ce sous-projet était le suivant : les recommandations alimentaires («mangez plus de poissons qui ne mangent pas d'autres poissons») s'étaient traduites par une baisse sensible de la contamination au mercure dans le village de Brasília Legal, mesurée dès le début des années 2000. Cependant, il avait été également remarqué que les fonctions visuelles (vision des couleurs et sensibilité aux contrastes) continuaient à baisser en fonction de l'exposition antérieure. Les chercheurs ont cherché à mesurer plus précisément ces atteintes aux fonctions visuelles. En octobre 2001, ils organisèrent donc un test ophtalmologique auprès de 98 habitants de Brasília Legal déjà inclus dans la recherche. Des optométristes de l'Université de Montréal installèrent un centre de consultation temporaire pour effectuer ces mesures. Il fallait distinguer entre les personnes qui avaient un mauvais résultat aux tests ophtalmologiques en raison de la contamination au mercure et celles qui échouaient en raison d'autres pathologies ou traumas oculaires. Un opticien était donc présent pour équiper les personnes présentant un défaut visuel non lié au mercure, afin que les résultats des tests ne soient pas contestables.

Dans le dispositif, il avait été prévu de procurer des lunettes aux gens examinés qui en auraient besoin. Les verres étaient déjà montés et réglés pour les niveaux de correction les plus courants. Tous les équipements optiques provenaient de donations d'entreprises canadiennes. Il y avait aussi des lunettes de soleil qui-sans que cela n'ait été apparemment collectivement décidé - étaient remises à « toutes les personnes examinées, avec ou sans prescription" (Piéraut, 2004a). Très vite, dans le village et dans les communautés autour de Brasília Legal, le bruit a couru que «l'équipe du projet mercure» organisait un diagnostic des problèmes de vision, prescrivait gratuitement des lunettes de vue à qui en aurait besoin et off rait des lunettes de soleil. De nombreuses personnes ont alors afflué depuis les autres villages de la région (souvent distants de plusieurs heures de canot). Chercheurs et optométristes ont été rapidement débordés par cet engouement. Ces derniers n'avaient pas prévu suffisamment de lunettes pour équiper tous ceux qui étaient venus.

Surtout, face à l'afflux de personnes se présentant au centre d'examen, il fallut faire des choix: les participants au Caruso étaient examinés en priorité puisqu'ils étaient suivis dans le cadre du projet, mais leur situation ophtalmologique pouvait être moins urgente que celle d'autres 
personnes venues éventuellement de communes ne participant pas au projet. Certains habitants avaient reçu des lunettes dont les corrections prédéfinies ne convenaient finalement pas ${ }^{7}$. Les lunettes de soleil causèrent la discorde entre les enfants de l'école et parmi les adultes. Des réunions publiques furent organisées lors desquelles les chercheurs, les leaders locaux et les habitants constatèrent qu'il nétait pas possible de satisfaire tout le monde. Le test fut conduit à son terme, mais l'épisode plongea chercheurs et communautés dans un certain désarroi.

En 2006, afin d'étudier plus précisément les facteurs en cause dans les troubles de la vision, les chercheurs ont entrepris un nouvel examen ophtalmologique. Le nombre de personnes incluses dans la recherche était alors bien plus important que la première fois (448), mais une bonne part d'entre elles avaient déjà collaboré à l'enquête sur les relations entre alimentation et niveau de mercure. L'examen concernait 12 communes (et non plus une) et se déroula sur une période de deux mois et demi. Deux navettes fluviales affrétées spécialement transportaient les personnes entre Itaituba et leurs habitations, situées en amont et en aval. Afin d'éviter de connaître le même débordement que la première fois, des réunions d'information publiques avaient été organisées en amont. Les habitants avaient été informés que les participants au projet de recherche se verraient proposer un examen ophtalmologique suivi d'une prise en charge de lunettes si l'examen décelait un besoin. Des optométristes canadiens y participèrent de nouveau, ainsi que deux assistants de recherche locaux. Là encore, pour ceux dont l'examen montrait l'existence d'un besoin de lunettes, monture et verre étaient pris en charge, mais c'était cette fois l'opticien d'Itaituba qui les montait ${ }^{8}$.

Ces précautions nont cependant pas suffi à éviter toutes les difficultés. Même si logistiquement l'opération s'est bien mieux déroulée qu'en 2001, les relations entre chercheurs et participants en ont été de nouveau troublées. Pour participer à l'examen, certaines personnes devaient faire les

7. Des témoins, chercheurs et habitants se rappellent que des personnes échangèrent entre elles des lunettes dont la prescription était différente et que la nuit, quelqu'un s'introduisit par effraction dans le laboratoire pour... échanger sa paire de lunettes.

8. Dans le récit qu'il a rédigé à son retour, l'opticien estimait qu'une meilleure solution aurait été d'avoir agi "non pas en "gringos", mais en missionnaire ", c'est-à-dire en transmettant son savoir-faire d'opticien (montage, réglage, etc.) à une personne sur place. Le choix qui a été fait en 2006 était de s'appuyer sur les ressources locales. 
trajets aller-retour de nuit et s'absenter pendant plus de trente-six heures de chez eux. Certains habitants, en raison de leur travail (agricole ou autre), n'ont pu se libérer pour participer à cet examen alors qu'ils en auraient eu besoin. Et à l'échelle régionale, des habitants de communes voisines qui ne participaient pas au projet ont trouvé injuste de ne pas avoir la possibilité de bénéficier, eux aussi, de cet examen utile. Lopération semble avoir généré là encore une certaine frustration parmi la population riveraine.

Au final, ces deux tests ophtalmologiques ont permis de générer des connaissances pertinentes concernant les effets du mercure sur la vision, mais ils semblent avoir constitué l'un des facteurs perturbateurs de la relation entre chercheurs et participants (incompréhensions, attentes déçues, ambiguités, etc.). En 2009, il arrivait encore que les habitants de la région du Tapajós associent l'équipe du «projet mercure » avec la possibilité d'obtenir des lunettes. Alors que les chercheurs leur rendaient visite sans mentionner le problème particulier des problèmes de vision, des habitants leur demandaient des comptes sur cette fourniture de lunettes, en insistant sur l'importance que cela représentait à leurs yeux. Les chercheurs répondaient, avec un certain embarras, ne pas pouvoir ou ne pas vouloir le faire.

\section{DEVOIRS DES CHERCHEURS ET PRISE EN CHARGE}

Quelles leçons peut-on tirer de ces deux épisodes? Il n'y a certes pas eu de grave problème éthique, mais il en est issu un trouble diffus, qui appelle plusieurs remarques. D'abord, il faut rappeler qu'aucune recherche au contact de la population n'est absolument anodine, sans conséquence sur la vie des gens. Toute recherche de terrain entraîne pour les chercheurs comme pour les participants des expériences singulières qui ne sont pas humainement neutres. De plus, ces opportunités ne sont pas nécessairement réparties équitablement. Le budget des chercheurs étant nécessairement limité, le nombre de participants l'est aussi, la recherche ne concerne alors qu'un sous-groupe d'une population qui peut d'ailleurs avoir été choisi par hasard, pour des raisons contingentes, «bêtement» pratiques. En l'occurrence, en 2001 c'est à Brasília Legal que le test avait été réalisé, car c'était dans ce village-là, desservi par des transports fluviaux réguliers, que l'équipe de recherche s'était initialement implantée. 
Le fait que la recherche s'accompagne de la délivrance d'un bien ou d'un service (ici, la prise en charge d'une correction optique) renforce l'inégalité entre les inclus et les non-inclus. En l'occurrence, cette action de diagnostic et de prise en charge s'est faite dans une région où les gens qui ont des problèmes de vue n'ont généralement pas les ressources nécessaires pour aller jusqu'à Itaituba ou Santarém et s'offrir des lunettes de vue. Dans le village de Brasília Legal, par exemple, il n'y a qu'un poste de santé auquel est affecté un seul agent de santé communautaire, qui doit se rendre dans tous les villages alentour sans équipement particulier ni service d'ophtalmologie.

La question est donc la suivante: pour une équipe de recherche étrangère, présente périodiquement dans la région, et n'intervenant qu'auprès d'une partie de la population régionale, faut-il voir cette situation de carence généralisée comme une raison de ne pas offrir de prise en charge ou, au contraire, comme une raison de proposer une prise en charge, même limitée dans sa portée et dans sa durée?

On peut considérer qu'il en va de la prise en charge comme du devoir d'information concernant les résultats des tests. De façon générale, dans le cadre du projet Caruso, les résultats des tests étaient restitués à la fois sous une forme individuelle à chaque participant et sous la forme de résultats généraux lors de réunions publiques. En principe, les participants savaient ce que les chercheurs savaient à leur propos. En outre, le diagnostic visuel à lui seul ne soulevait pas de problème éthique particulier (Tortevoye et al., 2007). Mais si le diagnostic montre chez le participant un problème de vue, et que des moyens, non disponibles localement, existent pour le corriger, même imparfaitement, est-ce un devoir pour les chercheurs de mettre en œuvre ces moyens?

Les textes de référence du Conseil des Organisations internationales des sciences médicales pour la recherche biomédicale évoquent le devoir, lorsque les recherches concernent des populations aux ressources limitées, d'assurer un «accès raisonnable» aux produits ou dispositifs testés ${ }^{9}$. Dans

9. La directive $\mathrm{n}^{\circ} 10$ des Lignes directrices internationales d'éthique pour la recherche biomédicale impliquant des sujets humains, édictées par le Conseil des Organisations internationales des sciences médicales (CIOMS), avec la collaboration de l'Organisation mondiale de la Santé (OMS), à Genève, en 2003, stipule que toute intervention ou tout produit mis au point ou toute connaissance obtenue sera raisonnablement mis à la disposition au bénéfice de cette population ou communauté. Cette 
le projet vision du Caruso, il n'est pas question de tester une méthode thérapeutique, mais bien d'une recherche visant à comprendre les effets du mercure et d'accompagner les habitants dans la définition de solutions robustes à ces effets. Le principe d'un «accès raisonnable » concerne donc ici les résultats de la recherche, à savoir les connaissances produites sur le système humain/environnement/mercure. Les chercheurs ont pourtant considéré qu'il était de leur responsabilité d'aller au-delà et d'assurer, pour les personnes ayant un problème de vue diagnostiqué au cours de la recherche, un accès à des services ophtalmologiques. En se positionnant, par l'acte de dépistage, du point de vue de la responsabilité clinique, individuelle - l'éthique du soin -, ils ont estimé qu'il était de leur devoir de mettre à disposition des lunettes pour ceux que cela aiderait.

Si l'on se replace du point de vue de l'éthique de la santé publique et du bien-être collectif, le raisonnement est différent. Par exemple, une mesure utile sur le plan individuel, mais qui s'avère collectivement injuste car inégalitaire, est-elle collectivement plus utile que la décision de s'abstenir? La réponse à cette question dépend de la «valeur » attribuée à l'injustice, ainsi que de la comparaison entre les deux faces de l'action réalisée : d'un côté, en fournissant des lunettes aux participants, les chercheurs ont probablement apporté un bénéfice net, direct, répondant à des besoins ciblés; d'un autre côté, cette intervention, en ne touchant pas au levier principal (l'offre de santé à l'échelle régionale), pourrait aussi avoir l'effet pervers commun aux actions d'assistance, soit se substituer temporairement à l'offre publique de soins, sans que cela conduise à mettre en place celle-ci. Faute de données factuelles, on ne tranchera pas ici cette question.

Il reste à aborder l'autre dimension sous-jacente à ce cas, celle de la compensation à la recherche. En ce qui concerne l'intention de l'équipe de chercheurs, deux versions existent. La première est donnée par exemple par l'opticien qui participait en 2001 à la recherche, qui qualifie de façon ambivalente la participation des habitants. D'un côté, la mise à disposition de lunettes est présentée comme faisant partie intégrante du protocole de

directive fait suite au débat à propos de la révision du paragraphe 30 de la déclaration d'Helsinki (Assemblée médicale mondiale) en 2000. La notion de disponibilité raisonnable, assez vague, laisse la porte ouverte à la négociation pour savoir qui, des chercheurs ou des pouvoirs publics, doit assurer cette mise à disposition des traitements à l'issue de l'essai, pour combien de temps, et qui peut y avoir droit (les participants seulement ou l'ensemble de la communauté ?). 
recherche, en tant qu'outil pour "capturer", en minimisant les biais, les troubles de la vision attribuables au mercure dans cette population. Dès lors, l'image est celle de chercheurs qui, animés d'une intention louable, mettent en place un test avec ses contraintes et ses avantages auprès de populations elles aussi promptes à coopérer : «En venant étudier leur problème, dit l'opticien, nous les avons beaucoup dérangés dans leur quotidien. Et pourtant (par curiosité peut-être?), ils se sont toujours montrés très coopératifs» (Piéraut, 2004b). D’un autre côté, les lunettes sont présentées comme un outil de fidélisation, un mécanisme de compensation, permettant de "garder la population intéressée à passer la batterie de tests [...]. Comme les tests visuels arrivaient en dernier, les lunettes données étaient en quelque sorte une récompense, un cadeau de participation comme dans les soirées bénéfices» (Piéraut, 2004a). Ces récompenses données aux participants sont donc présentées d'emblée comme "acceptables ${ }^{10}$, mais ce récit paraît désenchanter la relation entre chercheurs et participants souvent valorisée dans la recherche participative.

De fait, certains chercheurs interrogés se démarquent vivement de cette interprétation et en proposent une seconde : les lunettes nétaient en rien une compensation ou un cadeau, mais bien un geste sanitaire qui était éthiquement requis. Pour eux, prescrire et mettre à disposition des lunettes à qui en a besoin, c'est articuler convenablement devoirs et intérêts de chacun: d'un côté, certes les participants «donnent un peu de soi» (FagotLargeault, 1991), prennent du temps pour passer le test et contribuer à l'évaluation de la relation entre mercure et problèmes de vue; d'un autre côté, ils peuvent «bénéficier» non seulement d'un retour d'information sous la forme de résultats optométriques et de connaissances plus générales, mais aussi, le cas échéant, d'un accès immédiat à des lunettes pour corriger les difficultés repérées. Dans cette optique, on ne saurait établir de relation de réciprocité entre ces deux «dons ». Les intérêts de chacun - les

10. Le commentaire de la recommandation $n^{\circ} 7$ des Lignes directrices éthiques internationales pour la recherche épidémiologique du CIOMS (2009) pose en effet une distinction entre les récompenses « acceptables » et les récompenses «inacceptables ». Parmi les premières figurent le remboursement des frais, une somme d'argent modeste pour dédommager des contraintes non compensées par ailleurs, des prestations médicales (qu'elles soient ou non liées à la recherche) et l'accès gratuit à des procédures et à des tests. Les récompenses inacceptables sont celles qui, étant donné leur nature, le contexte ou la somme d'argent à laquelle elles correspondent, faussent le jugement du participant. Bien entendu, la limite entre les deux catégories est mouvante, mais l'idée générale est que des compensations peuvent être légitimes. 
chercheurs voulant obtenir des données et les habitants, bénéficier d'une prise en charge - ne sont pas séparés, mais mutuellement partagés.

De façon générale, dans le projet Caruso, le choix avait été fait d'éviter autant que possible que la participation des personnes se fasse en échange de contreparties en nature ou en argent. Si la recherche implique pour les participants des coûts directs (carburant, aliments, etc.), ceux-ci sont pris en charge par les chercheurs qui ont un budget pour cela. En revanche, les temps de réunion, de conversation, le temps passé pour répondre à des questionnaires ou des consultations ne sont pas rémunérés. On pourrait d'ailleurs considérer que, sur le principe, cette position n'est pas évidente. Pourquoi les chercheurs reçoivent-ils un salaire tandis que les participants n'en reçoivent pas? La sage-femme qui a coordonné le recueil de données d'alimentation auprès des femmes de son village a d'ailleurs justement été rémunérée, au titre de collaboratrice. Mais peut-être que si le salaire était généralisé à tous, le paiement du temps passé et la mise en place de relations salariales risqueraient de générer des perturbations sur l'économie locale et surtout de transformer la relation entre les chercheurs et les communautés en relation employeur-employé, qui risquerait de produire principalement des effets de dépendance.

Les témoignages recueillis indiquent que les chercheurs du Caruso, probablement ravis des relations qui se nouaient d'année en année avec les habitants et désireux de leur être directement bénéfiques, apportaient souvent avec eux des choses jugées utiles à la communauté (des médicaments listés par l'infirmière du village, des cahiers pour l'école, etc.). Ces choses leur permettaient de faire "un cadeau collectif » aux communautés qui les accueillaient, sans pour autant entrer dans un système d'achat de participation de chacun. Il semble que les lunettes, à la fois parce qu'elles constituaient un bien rare et précieux non disponible par ailleurs, parce quelles bénéficiaient à certains et non à tous et parce que leur mise à disposition était centrée sur l'individu et non sur le collectif, ont ébranlé cette approche collective et communautaire. 


\section{LES EXIGENCES DISTINCTES DE LA RECHERCHE ET DE L'ACTION}

Pour conclure, je développerai deux commentaires pour mettre en perspective ce cas. Le premier rappelle l'exigence - au demeurant très ambitieuse-de participation des communautés dans l'approche écosystémique. Quel bilan les communautés riveraines du Tapajós tirent-elles de ce dispositif? Jusquoù ont-elles été parties prenantes dans le montage assuré par les chercheurs pour réaliser des diagnostics d'acuité visuelle ? Il faut noter que ni en 2001 ni en 2006 les habitants nont été associés à la décision de proposer une prise en charge à l'issue du test de dépistage, ce qui explique probablement que les chercheurs, croyant bien faire, n'avaient pas envisagé toutes les conséquences de cette mesure. D'un autre point de vue, l'afflux de personnes de toute la région et le débordement du dispositif conçu par les chercheurs (notamment lors de la première occurrence du "projet vision », 2001) ne sont peut être pas tant le signe d'une maladresse dans la communication qu'une façon pour la réalité de se rappeler aux chercheurs. On peut regarder l'épisode optométrique et les remous qu'il a générés, non plus seulement comme une perturbation de la relation entre chercheurs et participants, mais de manière plus constructive comme une façon pour les habitants d'intervenir sur l'intervention des chercheurs. En réclamant des lunettes, les populations riveraines ont «participé » au projet des chercheurs en indiquant ce qui, pour elles, constituait un sujet de mobilisation possible. Il revenait alors aux chercheurs, en fonction de leurs possibilités et de leurs propres intérêts, de suivre ou non cette suggestion. En l'occurrence, le projet de recherche n'était pas centré sur la question de l'accès aux soins dans la région, mais sur celle de la contamination au mercure et des mesures de prévention primaire. L'approche globale ambitionnée par les chercheurs a probablement rendu plus difficile la démarcation entre ce qui relevait ou non de leur champ d'intervention. La "proposition" des habitants est pour ainsi dire restée sans réponse adéquate, même si c'est l'action des chercheurs qui l'a en quelque sorte suscitée.

Le second commentaire renvoie donc à la question du rôle des chercheurs et à la définition de leur sphère de responsabilité. L'introduction du rapport Belmont, citée plus haut, indique en substance que l'éthique de la recherche et l'éthique de la pratique sont deux sphères qui ne se 
recouvrent pas complètement. J'en proposerai l'interprétation suivante : les chercheurs ont des responsabilités particulières en tant que chercheurs parce que la recherche est, en quelque sorte, un «luxe». Produire des connaissances nouvelles n'est jamais une obligation prima facie, mais un choix qui se fait en fonction de buts et d'intérêts déterminés ${ }^{11}$. Chercher à générer des connaissances, c'est un supplément, préférable, utile si l'on veut, mais jamais une obligation en soi. C'est en ce sens que des exigences éthiques particulières ont été mises en place, propres aux recherches conduites avec les êtres humains (notamment les principes de respect, de bienfaisance et de justice). Il s'agit de garantir que la recherche, qui n'est donc jamais absolument requise, ne sera pas conduite en lésant des individus ou des groupes. Par contraste avec la recherche, conçue comme une activité qui pourrait aussi ne pas être, l'action - qu'elle soit médicale, humanitaire, individuelle, etc. - est en prise avec des exigences nées de conditions d'existence. Intervenir dans et sur le monde ne relève pas d'un choix, c'est une condition attachée à la vie humaine. Il ne s'agit pas de dire qu'il n'existe pas d'actions superflues, ni que les actions ne devraient pas être soumises à l'évaluation éthique. Léthique de la pratique suppose évidemment d'interroger le bien-fondé des intentions, les différentes conséquences et d'envisager les différentes options possibles - y compris celle de ne pas agir. Simplement, garder à l'esprit la distinction entre recherche et action permet d'éviter de confondre deux registres de justifications distincts.

Comme on l'a vu au début de cet article, les RPC ont pour particularité de se tenir simultanément dans les deux sphères. Cette ambivalence génère des ambiguiités auxquelles font face les chercheurs aussi bien que les communautés. Elles se tiennent entre ce qui est de l'ordre du possible (la recherche) et ce qui est de l'ordre de l'inévitable (l'action). Les RPC ne sont ni simplement recherche, se tenant à bonne distance de leur objet, ni simplement action, en prise à un contexte dans lequel elles sont engagées. Loscillation entre recherche et action, distance et participation ne semble vertueuse que dans la mesure où elle s'accompagne d'un surcroît de vigilance et de lucidité.

11. Tout programme de recherche s'inscrit dans un schème de signification, un ensemble d'intérêts et de buts qui ne s'imposent pas d'eux-mêmes et requièrent donc d'être explicités et discutés. C'est ce que rappelle clairement Kitcher (2010). 


\section{BIBLIOGRAPHIE}

Adelman, C. (1993), "Kurt Lewin and the origins of action research », Educational Action Research, vol. 1, no 1, p. 7-24.

Chamayou, G. (2008), Les corps vils. Expérimenter sur les êtres humains aux XVIII et XIX siècles, Paris, La Découverte.

COMmission nATIONALE AMÉRICAIne (1984), "Rapport Belmont », Cabiers de bioéthique. Médecine et expérimentation, $\mathrm{n}^{\circ} 4$, (traduction fr. légèrement modifiée).

Dansereau, P. (2003), "Préface », dans J. LEBEL, La santé: une approche écosystémique, Ottawa, Centre de recherches pour le développement international, p. 8.

Fagot-Largeault, A. (1991), «Autonomie, don et partage dans la problématique de l'expérimentation humaine», Dialogue, vol. 30, n 3 , p. 355363.

Freire, P. (1974), Pédagogie des opprimés, suivi de Conscientisation et révolution, Paris, F. Maspéro.

GASPAR, E. (2003), «Contamination mercurielle en Amazonie : recherche participative avec des femmes riveraines pour réduire l'exposition au mercure». Thèse de doctorat en sciences de l'environnement, Montréal, Université du Québec à Montréal. Thèse non soutenue.

Green, L., M. Daniel et L. Novick (2001), «Partnerships and coalitions for community-based research », Public Health Reports, no 116, suppl. 1, p. 2031.

ISRAEL, B., et al. (1994), "Health education and community empowerment: conceptualizing and measuring perceptions of individual, organizational and community control », Health Education Quarterly, vol. 21, n 2, p. 149170 .

ISRAEL, B., et al. (1998), «Review of community-based research : Assessing partnership approaches to improve public health", Annual Review Public Health, no 19 , p. 173-202.

King, N, G. Henderson et J. STEIN (1999), «Beyond regulations: Ethics in human subjects research», dans N. KING, G. HENDERSON et J. STEIN (dir.), Chapel Hill, North Carolina, University of North Carolina Press, p. 22-24.

KiTcher, Philip (2010), Science, vérité, démocratie, Paris, Presses universitaires de France. 
LACEy, H. (2005), Is Science Value Free? Values and Scientific Understanding, London/New York, Routledge.

LEBEL, J. (2002), «Hommage à un chercheur brésilien, Fernando Branches ", texte initialement distribué par courriel, [En ligne], [http:// www.idrc.ca/fr/ev-29405-201-1-DO_TOPIC.html], (12 février 2010).

LeBel, J. (2003), La santé: une approche écosystémique, Ottawa, Centre de recherches pour le développement international.

LEWIN, K. (1946), "Action research and minority problems », Journal of Social Issues, vol. 2, $\mathrm{n}^{\circ} 4$, p. 34-46.

LucotTe, M., et al. (2005), «Human exposure to mercury as a consequence of landscape management and socioeconomical behaviors. Part I: the Brazilian Amazon case study", $R M Z-$ Materials and Geoenvironment, $\mathrm{n}^{\circ}$ 51, p. $668-672$.

Macklin, R. (1999), Against Relativism: Cultural Diversity and the Search for Ethical Universals in Medicine, New York, Oxford University Press.

MAssé, R. (2003), «Valeurs universelles et relativisme culturel en recherche internationale: les contributions d'un principisme sensible aux contextes socioculturels", Autrepart. Revue de l'Institut de recherche pour le développement (IRD), no 28, p. 21-35.

MERTENS, et al. (2005), «A network approach for analysing and promoting equity in participatory ecohealth research ", EcoHealth, vol. 2, n 2, p. 113126.

Minkler, M., et al. (2003), "Community-based participatory research : implications for public health funding", American Journal of Public Health, vol. 93, no 8, p. 1210.

MinkleR, M. (2004), "Ethical challenges for the "outside" researcher in community-based participatory research", Health Education E Behavior, vol. 31, n 6, p. 684-701.

Mohan, G. (1999), «Not so distant, not so strange: The personal and the political in participatory research », Ethics, Place and Environment, vol. 2, $\mathrm{n}^{\mathrm{o}} 1$, p. 41-54.

Muller-Mirza, N. (2009), "Qu'est-ce qui se développe dans et par une formation-action ?», dans P. BEGUIN et M. CERF (dir.), Dynamique des savoirs, dynamique des changements, Toulouse, Octares, p. 229-244.

Ouellet-Dubé, F. (1979), «Recherche ou pratique : qui gagne? », Service social, vol. $28, \mathrm{n}^{\circ} 2$, p. 5-14. 
Passos, C.J.S., et D. Mergler (2005), « Human mercury exposure and adverse health effects in the Amazon: a review ", Cadernos de Saúde Pública, Rio de Janeiro, $\mathrm{n}^{\circ} 24$, supl. 4, p. 503-520.

PAssos, C.J.S. (2009), "Alimentation locale et exposition au mercure en Amazonie brésilienne», Bulletin d'information en santé environnementale, $\mathrm{n}^{\mathrm{o}} 20$, p. $10-14$.

PiÉraut, J. (2004a), «Un opticien québécois en Amazonie, 3e partie», Coup d'œil, [En ligne], [http://www.bretoncom.net/coupdoeil/2004/03/10.asp], (22 février 2010).

PiÉRAUT, J. (2004b), «Un opticien à Brasília Legal», récit publié sur le site du Forum amazonien sur la contamination des écosystèmes par le mercure (1999-2004), [En ligne], [http://www.facome.uqam.ca/pdf/Un_opticien _a_Brasilia_Legal.PDF], (20 février 2010).

Roulet, M., et al. (1999), "Effects of recent human colonization on the presence of mercury in Amazonian ecosystems ", Water, Air and Soil Pollution, $\mathrm{n}^{\circ} 112$, p. 297-313.

SHORE, N. (2006), «Re-conceptualizing the Belmont report: A communitybased participatory research perspective ", Journal of Community Practice, vol. $14, \mathrm{n}^{\circ} 4$, p. 5-26.

Strauss, L.A., et J. Corbin (2008), Basics of Qualitative Research: Techniques and Procedures for Developing Grounded Theory, Londres, Sage.

Thiery, O., et M. Cerf (2009), «Penser la recherche participative comme une pratique. Une proposition de diagnostic », dans P. BEGUIN et M. CERF (dir.), Dynamique des savoirs, dynamique des changements, Toulouse, Octares, p. 44.

Tortevoye, P., et al. (2007), "Analyse des enjeux éthiques soulevés au cours d'un programme de recherche épidémiologique de dix années en Guyane française: limites de l'encadrement actuel et solutions adoptées », Revue d'épidémiologie et de santé publique, $\mathrm{n}^{\circ}$ 55, p. 413-21.

Turckheim, E., B. HuberT et M. Cerf (2009), «L'évaluation des recherches partenariales: quelle procédure, quels critères?", dans P. BEGUIN et M. CERF (dir.), Dynamique des savoirs, dynamique des changements, Toulouse, Octares. 